\title{
A bijection between (bounded) dominant Shi regions and core partitions
}

\author{
Susanna Fishel ${ }^{1}$ and Monica Vazirani ${ }^{2}$ \\ ${ }^{1}$ School of Mathematical and Statistical Sciences, Arizona State University, Tempe, AZ 85287-1804, USA \\ ${ }^{2}$ University of California, Davis, Department of Mathematics, One Shields Ave, Davis, CA 95616-8633, USA
}

\begin{abstract}
.
It is well-known that Catalan numbers $C_{n}=\frac{1}{n+1}\left(\begin{array}{c}2 n \\ n\end{array}\right)$ count the number of dominant regions in the Shi arrangement of type $A$, and that they also count partitions which are both $n$-cores as well as $(n+1)$-cores. These concepts have natural extensions, which we call here the $m$-Catalan numbers and $m$-Shi arrangement. In this paper, we construct a bijection between dominant regions of the $m$-Shi arrangement and partitions which are both $n$-cores as well as $(m n+1)$-cores.

We also modify our construction to produce a bijection between bounded dominant regions of the $m$-Shi arrangement and partitions which are both $n$-cores as well as $(m n-1)$-cores. The bijections are natural in the sense that they commute with the action of the affine symmetric group.

\section{Résumé.}

Il est bien connu que les nombres de Catalan $C_{n}=\frac{1}{n+1}\left(\begin{array}{c}2 n \\ n\end{array}\right)$ comptent non seulement le nombre de régions dominantes dans le Shi arrangement de type $A$ mais aussi les partitions qui sont à la fois $n$-coeur et $(n+1)$-coeur. Ces concepts ont des extensions naturelles, que nous appelons ici les nombres $m$-Catalan et le $m$-Shi arrangement. Dans cet article, nous construisons une bijection entre régions dominantes du $m$-Shi arrangement et les partitions qui sont à la fois $n$-coeur et $(n m+1)$-coeur.

Nous modifions également notre construction pour produire une bijection entre régions dominantes bornées du $m$-Shi arrangement et les partitions qui sont à la fois $n$-coeur et $(m n-1)$-coeur. Ces bijections sont naturelles dans le sens où elles commutent avec l'action du groupe affine symétrique.
\end{abstract}

Keywords: cores, symmetric group, Shi arrangement, Catalan numbers

\section{Introduction}

Let $\Delta$ be the root system of type $A_{n-1}$, with Weyl group $W$, and let $m$ be a positive integer. Then let $\mathcal{S}_{n}^{m}$ be the arrangement of hyperplanes $H_{\alpha, k}=\left\{x \mid\langle\alpha \mid x\rangle=k\right.$ for $-m+1 \leq k \leq m$ and $\left.\alpha \in \Delta^{+}\right\}$. $\mathcal{S}_{n}^{m}$ is the $m$ th extended Shi arrangement of type $A_{n-1}$, called here the $m$-Shi arrangement.

In Fishel and Vazirani (2010, 2009), the authors constructed and analyzed bijections between certain regions of $\mathcal{S}_{n}^{m}$ and certain $n$-cores. In this extended abstract, we summarize the results from both papers. We will first construct and discuss a bijection between dominant regions of $\mathcal{S}_{n}^{m}$, and partitions that are 
$n$-cores as well as $(m n+1)$-cores. We will then modify the construction to give a direct bijection between bounded dominant regions and partitions which are simultaneously $n$-cores and $(m n-1)$-cores.

Our bijection is $W$-equivariant in the following sense. In each connected component of $\mathcal{S}_{n}^{m}$ there is exactly one $m$-minimal alcove, the alcove closest to the fundamental alcove $\mathcal{A}_{0}$, and in each bounded connected component there is exactly one $m$-maximal alcove, the alcove farthest from the fundamental alcove $\mathcal{A}_{0}$. Since the affine Weyl group $W$ acts freely and transitively on the set of alcoves, there is a natural way to associate an element $w \in W=\widehat{\mathfrak{S}}_{n}$ to any alcove $w^{-1} \mathcal{A}_{0}$, and to the $m$-minimal and $m$-maximal alcoves in particular. There is also a natural action of $\widehat{\mathfrak{S}}_{n}$ on partitions, whereby the orbit of the empty partition $\emptyset$ is precisely the $n$-cores. We will show that when $w$ is associated to an $m$-minimal alcove, then $w \emptyset$ is an $(m n+1)$-core as well as an $n$-cores and that all such $(m n+1)$-cores that are also $n$-cores can be obtained this way. We will also show the analogous result for $m$-maximal alcoves and $(m n-1)$-cores that are also $n$-cores.

Roughly speaking, to each $n$-core $\lambda$ we can associate an integer vector $\vec{n}(\lambda)$ whose entries sum to zero, as in Garvan et al. (1990). When $\lambda$ is also an $(m n+1)$-core, these entries satisfy certain inequalities. On the other hand, these are precisely the inequalities that describe when a dominant alcove is $m$-minimal. We $\lambda$ is an $(m n-1)$-core, the inequalites which must be satisfied by the entries of the vector exactly describe when a dominant alcove is $m$-maximal.

As a consequence, we show an $n$-core $\lambda$ is automatically an $(m n+1)$-core if $\varepsilon_{i}(\lambda) \leq m$ for all $0 \leq i<n$, where $\varepsilon_{i}(\lambda)$ counts the number of removable boxes of residue $i$. We also show the related result, that an $n$-core $\lambda$ is automatically an $(m n-1)$-core if $\varphi_{i}(\lambda) \leq m$ for all $0 \leq i<n$, where $\varphi_{i}(\lambda)$ counts how many addable boxes of residue $i$ the partition $\lambda$ has.

The article is organized as follows. In Section 2 we introduce notation and recall facts about Coxeter groups, root systems of type $A$, and inversion sets for elements of the affine symmetric group. Section 3 explains how the position of $w^{-1} \mathcal{A}_{0}$ relative to our system of affine hyperplanes is captured by the action of $w$ on affine roots and that $m$-minimality and $m$-maximality can each be expressed by certain inequalities on the entries of $w(0,0, \ldots, 0)$. In Section 4 we review facts about core partitions and in particular remind the reader how to associate an element of the root lattice to each core. Our main theorems, the bijection between dominant regions of the $m$-Shi arrangement and special cores and the bijection between bounded dominant regions of the $m$-Shi arrangement and other special cores, is in Section 5 . Section 6 describes the effect of a related bijection on $m$-minimal and $m$-maximal alcoves. In Section 7 , we derive further results that refine our bijection between alcoves and cores and that involve Narayana numbers. We also characterize alcove walls in terms of addable and removable boxes.

\section{Preliminaries}

Please also see Fishel and Vazirani (2010, 2009). Let $\Delta$ be the root system for type $A_{n-1}$, with Weyl group the symmetric group $\mathfrak{S}_{n}$. Let $\widetilde{\Delta}$ be the affine root system of type $A_{n-1}^{(1)}$, with null root $\delta$, and with Weyl group the affine symmetric group $\widehat{\mathfrak{S}}_{n}$. See $\operatorname{Kac}(1990)$ for more details. $\Delta$ spans a Euclidean space $V$ with inner product $\langle\mid\rangle$. Let $Q \subseteq V$ denote the root lattice for $\Delta$. Let $m$ be a positive integer. The $m$-Shi arrangement is the collection of hyperplanes

$$
\mathcal{S}_{n}^{m}=\left\{H_{\alpha, k} \mid \alpha \in \Delta^{+},-m<k \leq m\right\},
$$

where $H_{\alpha, k}=\{v \in V \mid\langle v \mid \alpha\rangle=k\}$. This arrangement can be defined for all types; here we are concerned with type $A$. 
The arrangement dissects $V$ into connected components we call regions. We refer to regions which are in the dominant chamber of $V$ as dominant regions. Each connected component of $V \backslash \bigcup_{\substack{\alpha \in \Delta^{+} \\ k \in \mathbb{Z}}} H_{\alpha, k}$ is called an alcove and the fundamental alcove is denoted $\mathcal{A}_{0}$.

We denote the (closed) half spaces $H_{\alpha, k}^{+}=\{v \in V \mid\langle v \mid \alpha\rangle \geq k\}$ and $H_{\alpha, k}^{-}=\{v \in V \mid\langle v \mid \alpha\rangle \leq k\}$. Note $\mathcal{A}_{0}$ is the interior of $H_{\theta, 1}^{-} \cap \bigcap_{i=1}^{n-1} H_{\alpha_{i}, 0}^{+}$and the dominant chamber is $\bigcap_{i=1}^{n-1} H_{\alpha_{i}, 0}^{+}$.

The affine symmetric group $\widehat{\mathfrak{S}}_{n}$ acts on $V$ (preserving $Q$ ) via affine linear transformations, and acts freely and transitively on the set of alcoves. We thus identify each alcove $\mathcal{A}$ with the unique $w \in \widehat{\mathfrak{S}}_{n}$ such that $\mathcal{A}=w \mathcal{A}_{0}$. We also note that we may express any $w \in \widehat{\mathfrak{S}}_{n}$ as $w=u t_{\gamma}$ for unique $u \in \mathfrak{S}_{n}, \gamma \in Q$, or equivalently $w=t_{\gamma^{\prime}} u$ where $\gamma^{\prime}=u(\gamma)$. If we embed $V$ into $\mathbb{R}^{n}$ by mapping $\alpha_{i}$ to $\varepsilon_{i}-\varepsilon_{i+1}$, note that $\gamma^{\prime}=w(0, \ldots, 0)$.

We also remind the reader that when $w^{-1}$ is a minimal length right coset representative for $\widehat{\mathfrak{S}}_{n} / \mathfrak{S}_{n}$, then we may write $w^{-1}=t_{\gamma^{\prime}} u$ where $u \in \mathfrak{S}_{n}$ and $\gamma^{\prime}$ is in the dominant chamber.

For $w \in \widehat{\mathfrak{S}}_{n}$, we define the inversion set $\operatorname{Inv}(w)=\left\{\alpha \in \widetilde{\Delta^{+}} \mid w(\alpha) \in \widetilde{\Delta^{-}}\right\}$. Notice that the length $\ell(w)=|\operatorname{Inv}(w)|$ for $w \in \widehat{\mathfrak{S}}_{n}$ is just the minimal number of affine hyperplanes separating $w^{-1} \mathcal{A}_{0}$ from $\mathcal{A}_{0}$. We will need the following well-known proposition and corollary, both describing $\operatorname{Inv}(w)$ and both proved in Fishel and Vazirani (2010).

Proposition 2.1. Let $w \in \widehat{\mathfrak{S}}_{n}$ and $\alpha+k \delta \in \widetilde{\Delta^{+}}$. Then $\alpha+k \delta \in \operatorname{Inv}(w)$ iff $w^{-1} \mathcal{A}_{0} \subseteq H_{-\alpha, k}^{+}$

Corollary 2.2. Suppose $w$ is a minimal length left coset representative for $\widehat{\mathfrak{S}}_{n} / \mathfrak{S}_{n}$. Then $\operatorname{Inv}(w)$ consists only of roots of the form $-\alpha+k \delta, k \in \mathbb{Z}_{>0}, \alpha \in \Delta^{+}$. Further, if $-\alpha+k \delta \in \operatorname{Inv}(w)$ and $k>1$ then $-\alpha+(k-1) \delta \in \operatorname{Inv}(w)$.

\section{3 m-minimal and $m$-maximal alcoves}

We say an alcove $w \mathcal{A}_{0}$ is $m$-minimal if it is the unique alcove in its region such that $\ell(w)$ is smallest. Such alcoves are termed "representative alcoves" by Athanasiadis. We can identify each connected component of the complement of the $m$-Shi arrangement with its unique $m$-minimal alcove.

If the region is bounded, we can also identify it with the unique alcove $w^{\prime} \mathcal{A}_{0}$ contained in it such that $\ell\left(w^{\prime}\right)$ is largest. In this situation we will say the alcove $w^{\prime} \mathcal{A}_{0}$ is $m$-maximal. Note that for unbounded regions, no such alcove exists.

See Figure 5 below for a picture of the $m$-maximal alcoves of type $A_{2}$ for $m=1,2$.

The following proposition is useful. For a given alcove, it characterizes the affine hyperplanes containing its walls and which simple reflections flip it over those walls (by the right action). It can be found in Shi (1987) in slightly different notation.

Proposition 3.1. Suppose $w \mathcal{A}_{0} \subseteq H_{\alpha, k}^{+}$but $w s_{i} \mathcal{A}_{0} \subseteq H_{\alpha, k}^{-}$

1. Then $w\left(\alpha_{i}\right)=\alpha-k \delta$.

2. Let $\beta=w^{-1}(0, \ldots, 0) \in V$. Then $\left\langle\beta \mid \alpha_{i}\right\rangle=-k$.

Using the coordinates of $V \subseteq \mathbb{R}^{n}$, we note $k=\gamma_{u(i)}-\gamma_{u(i+1)}$, where $w=t_{\gamma} u$.

Remark 3.2. Note, if $w \mathcal{A}_{0}$ is m-minimal, then whenever $k \in \mathbb{Z}_{\geq 0}$ and $w \mathcal{A}_{0} \subseteq H_{\alpha, k}^{+}$but $w s_{i} \mathcal{A}_{0} \subseteq H_{\alpha, k}^{-}$ then we must have $k \leq m$ in the case $\alpha>0$ and $k \leq m-1$ in the case $\alpha<0$. 
It is easy to see that this condition is not only necessary but sufficient to describe when $w \mathcal{A}_{0}$ is $m$ minimal. Together with Proposition 2.1. Proposition 3.1 says that when $\alpha_{i} \in \operatorname{Inv}(w)$ and $w\left(\alpha_{i}\right)=\alpha-k \delta$ then $k \leq m$, and for $\beta=w^{-1}(0, \ldots, 0)$ that $\left\langle\beta \mid \alpha_{i}\right\rangle \geq-m$.

Applying Remark 3.2 to positive $\alpha$ and alcoves in the dominant chamber, we get the following corollary.

Corollary 3.3. Suppose $w \mathcal{A}_{0}$ is in the dominant chamber and m-minimal.

1. If $w \mathcal{A}_{0} \subseteq H_{\alpha, k}^{+}$but $w s_{i} \mathcal{A}_{0} \subseteq H_{\alpha, k}^{-}$for some $\alpha \in \Delta^{+}, k \in \mathbb{Z}_{\geq 0}$, then $k \leq m$.

2. Let $\beta=w^{-1}(0, \ldots, 0)$. Then $\left\langle\beta \mid \alpha_{i}\right\rangle \geq-m$, for all $i$, and in particular $\langle\beta \mid \theta\rangle \leq m+1$.

Proof. The first statement follows directly from Proposition 3.1 and Remark 3.2 . To conclude that the second statement holds for all $i$, note that if $k \leq 0$ then automatically $k \leq m$.

It is possible to make a remark analogous to Remark 3.2 for the case of $m$-maximal alcoves and we derive a corollary analogous to Corollary 3.3

Corollary 3.4. Suppose $w \mathcal{A}_{0}$ is in the dominant chamber and m-maximal.

1. If $w \mathcal{A}_{0} \subseteq H_{\alpha, k}^{-}$but $w s_{i} \mathcal{A}_{0} \subseteq H_{\alpha, k}^{+}$for some $\alpha \in \Delta^{+}, k \in \mathbb{Z}_{\geq 0}$, then $k \leq m$.

2. Let $\beta=w^{-1}(0, \ldots, 0)$. Then $\left\langle\beta \mid \alpha_{i}\right\rangle \leq m$, for all $i$, and in particular $\langle\beta \mid \theta\rangle \geq-m+1$.

\section{Core partitions and their abacus diagrams}

In this section we review some well-known facts about $n$-cores and review the useful tool of the abacus construction. Details can be found in James and Kerber (1981).

There is a well-known bijection $\mathcal{C}:\{n$-cores $\} \rightarrow Q$ that commutes with the action of $\widehat{\mathfrak{S}}_{n}$. One can use the $\widehat{\mathfrak{S}}_{n}$-action to define the bijection, or describe it directly from the combinatorics of partitions via the work of Garvan-Kim-Stanton's $\vec{n}$-vectors in Garvan et al. (1990) or of Lascoux (2001), or as described in terms of balanced abaci as in Berg et al. (2009). Here, we will recall the description from Berg et al. (2009) as well as remind the reader of the $\mathfrak{S}_{n}$-action on $n$-cores.

We identify a partition $\lambda=\left(\lambda_{1}, \ldots, \lambda_{r}\right)$ with its Young diagram, the array of boxes with coordinates $\left\{(i, j) \mid 1 \leq j \leq \lambda_{i}\right\}$. We say the box $(i, j) \in \lambda$ has residue $j-i \bmod n$, and in that case, we often refer to it as a $(j-i \bmod n)$-box. Its hook length $h_{(i, j)}^{\lambda}$ is $1+$ the number of boxes to the right of and below $(i, j)$.

An $n$-core is a partition $\lambda$ such that $n \nmid h_{(i, j)}^{\lambda}$ for all $(i, j) \in \lambda$.

We say a box is removable from $\lambda$ if its removal results in a partition. Equivalently its hook length is 1 . A box not in $\lambda$ is addable if its union with $\lambda$ results in a partition.

Claim 4.1. Let $\lambda$ be an n-core. Suppose $\lambda$ has a removable $i$-box. Then it has no addable $i$-boxes. Likewise, if $\lambda$ has an addable $i$-box it has no removable $i$-boxes.

$\widehat{\mathfrak{S}}_{n}$ acts transitively on the set of $n$-cores as follows. Let $\lambda$ be an $n$-core. Then

$$
s_{i} \lambda= \begin{cases}\lambda \cup \text { all addable } i \text {-boxes } & \exists \text { any addable } i \text {-box, } \\ \lambda \backslash \text { all removable } i \text {-boxes } & \exists \text { any removable } i \text {-box, } \\ \lambda & \text { else. }\end{cases}
$$

It is easy to check $s_{i} \lambda$ is an $n$-core. 


\subsection{Abacus diagrams}

We can associate to each partition $\lambda$ its abacus diagram. When $\lambda$ is an $n$-core, its abacus has a particularly nice form, and then can be used to construct an element of $Q$. Each partition $\lambda=\left(\lambda_{1}, \ldots, \lambda_{r}\right)$ is determined by its hook lengths in the first column, the $\beta_{k}=h_{(k, 1)}^{\lambda}$.

An abacus diagram is a diagram, with entries from $\mathbb{Z}$ arranged in $n$ columns labeled $0,1, \ldots, n-1$, called runners. The horizontal cross-sections or rows will be called levels and runner $k$ contains the entry labeled by $r n+k$ on level $r$ where $-\infty<r<\infty$. We draw the abacus so that each runner is vertical, oriented with $-\infty$ at the top and $\infty$ at the bottom, and we always put runner 0 in the leftmost position, increasing to runner $n-1$ in the rightmost position. Entries in the abacus diagram may be circled; such circled elements are called beads. Entries which are not circled will be called gaps. We shall say two abaci are equivalent if they differ by adding a constant to all entries. Note, in this case we must cyclically permute the runners so that runner 0 is leftmost. Given a partition $\lambda$ its abacus is any abacus diagram equivalent to the one obtained by placing beads at entries $\beta_{k}=h_{(k, 1)}^{\lambda}$ and all $j \in \mathbb{Z}_{<0}$.

Remark 4.2. It is well-known that $\lambda$ is an n-core if and only if its abacus is flush, that is to say whenever there is a bead at entry $j$ there is also a bead at $j-n$.

We define the balance number of an abacus to be the sum over all runners of the largest level in that runner which contains a bead. We say that an abacus is balanced if its balance number is zero. Note that there is a unique abacus which represents a given $n$-core $\lambda$ for each balance number. Given a flush abacus, that is, the abacus of an $n$-core $\lambda$, we can associate to it the vector whose $i^{\text {th }}$ entry is the largest level in runner $i-1$ which contains a bead. The sum of the entries in this vector is the balance number of the abacus. When the abacus is balanced, we will call this vector $\vec{n}(\lambda)$, in keeping with the notation of Garvan et al. (1990). We note that $\vec{n}(\lambda) \in Q$, when we identify $Q$ with $\left\{\left(a_{1}, \ldots, a_{n}\right) \in \mathbb{Z}^{n} \mid \sum_{i} a_{i}=0\right\}$.

We recall the following claim, which can be found in Berg et al. (2009).

Claim 4.3. The map $\lambda \mapsto \vec{n}(\lambda)$ is an $\widehat{\mathfrak{S}}_{n}$-equivariant bijection $\{n$-cores $\} \rightarrow Q$.

We recall here results of Anderson (2002), which describe the abacus of an $n$-core that is also a $t$-core, for $t$ relatively prime to $n$. When $t=m n-1$, this takes a particularly nice form.

Proposition 4.4 (Anderson). Let $\lambda$ be an $n$-core. Suppose t is relatively prime to $n$. Let $M=n t-n-t$. Consider the grid of points $(x, y) \in \mathbb{Z} \times \mathbb{Z}$ with $0 \leq x \leq n-1,0 \leq y$ labelled by $M-x t-y n$. Circle a point in this grid if and only if its label is obtained from the first column hooklengths of $\lambda$ or its label is in $\mathbb{Z}_{<0}$. Then $\lambda$ is a t-core if and only if the following three conditions hold.

1. All beads in the abacus of $\lambda$ are at entries $\leq M$, in other words at $(x, y)$ with $0 \leq x \leq n-1$, $0 \leq y$

2. The circled points in the grid are upwards flush, in other words if $(x, y)$ is circled, so is $(x, y-1)$;

3. The circled points in the grid are flush to the right, in other words if $(x, y)$ is circled and $x \leq n-2$, so is $(x+1, y)$.

Note that the columns of this grid are exactly the runners of $\lambda$ 's abacus, written out of order, with each runner shifted up or down relative to its new left neighbor. The runners have also been truncated, which is irrelevant given condition (1) above. This shifting is performed exactly so labels in the same row are congruent $\bmod t$. This explains why the circles must be flush to the right as well as upwards flush. 
We will now analyze the special cases $t=m n+1$ and $t=m n-1$ to derive the conditions for a $n$-core to be a $m n \pm 1$-core.

Corollary 4.5. Let $\lambda$ be an n-core.

1. Then $\lambda$ is an $(m n+1)$-core if and only if $\left\langle\vec{n}(\lambda) \mid \alpha_{i}\right\rangle \geq-m$ for $0<i<n$ and $\langle\vec{n}(\lambda) \mid \theta\rangle \leq m+1$.

2. Let $\lambda$ be an $n$-core. Then $\lambda$ is an $(m n-1)$-core if and only if $\left\langle\vec{n}(\lambda) \mid \alpha_{i}\right\rangle \leq m$ for $0<i<n$ and $\langle\vec{n}(\lambda) \mid \theta\rangle \geq-m+1$.

Proof. In the notation of Proposition 4.4 in the special case $t=m n+1$, the columns of the grid are the runners of $\lambda$ 's abacus, written in reverse order. Furthermore, each runner has been shifted $m$ units down relative to its new left neighbor. So the condition of being flush to the right on Anderson's grid is given by requiring on the abacus that if the largest circled entry on runner $i+1$ is at level $r$ then runner $i$ must have a circled entry at level $r-m$. In other words, if $\left(a_{1}, \ldots, a_{n}\right)=\vec{n}(\lambda)$, then we require $a_{i}+m-a_{i+1} \geq 0$, i.e. $\left\langle\vec{n}(\lambda) \mid \alpha_{i}\right\rangle \geq-m$ for $0<i<n$. Recall the $0^{\text {th }}$ and $(n-1)^{\text {st }}$ and runners must also have this relationship (adding a constant to all entries in the abacus cyclically permutes the runners). This condition becomes $a_{n}+1+m-a_{1} \geq 0$, i.e. $\langle\vec{n}(\lambda) \mid \theta\rangle \leq m+1$.

In the other special case, $t=m n-1$, the columns of the grid are now the runners of $\lambda$ 's abacus, cyclically shifted so the 0-runner is now rightmost versus leftmost. Otherwise, the analysis is the same.

\section{The bijection between cores and alcoves}

Let $\Phi$ be the map

$$
\begin{aligned}
\{n \text {-cores }\} & \rightarrow\{\text { alcoves in the dominant chamber }\} \\
w \emptyset & \mapsto w^{-1} \mathcal{A}_{0},
\end{aligned}
$$

which is $\widehat{\mathfrak{S}}_{n}$-equivariant, except for the minor technicality that the action on cores is a left action, but we take the right action on alcoves when discussing the Shi arrangement.

Theorem 5.1. The map $\Phi: w \emptyset \mapsto w^{-1} \mathcal{A}_{0}$ for $w$ a minimal length left coset representative of $\widehat{\mathfrak{S}}_{n} / \mathfrak{S}_{n}$ induces a bijection between the set of $n$-cores that are also $(m n+1)$-cores and the set of m-minimal alcoves, which are in the dominant chamber of $V$.

Theorem 5.2. The map $\Phi: w \emptyset \mapsto w^{-1} \mathcal{A}_{0}$ for $w$ a minimal length left coset representative of $\widehat{\mathfrak{S}}_{n} / \mathfrak{S}_{n}$ induces a bijection from the set of $n$-cores that are also $(m n-1)$-cores to the set of m-maximal alcoves in the dominant chamber.

The first bijection is pictured below in Figure 1 and the second in Figure 2

\section{A bijection on alcoves}

Although they are not an ingredient in the main theorem of this paper, the following theorems build on the work of Section 3 They describe what the bijection $w \mathcal{A}_{0} \mapsto w^{-1} \mathcal{A}_{0}$ does to the $m$-minimal and $m$-maximal alcoves. In particular, we do not limit ourselves to dominant alcoves. 

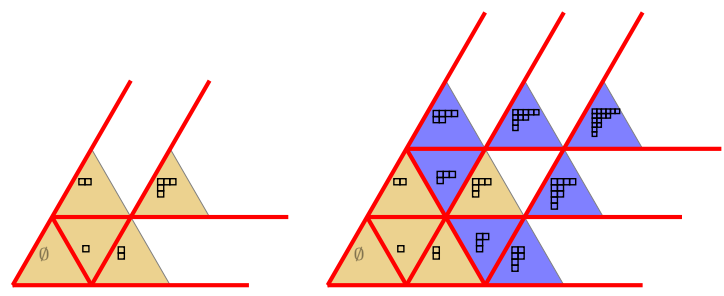

Fig. 1: $m$-minimal alcoves $w^{-1} \mathcal{A}_{0}$ in the dominant chamber of the $m$-Shi arrangement of type $A_{2}$, filled with the 3 -core partition $w \emptyset$. On the left $(m=1)$, they are also 4-cores, and on the right $(m=2)$, they are also 7 -cores.
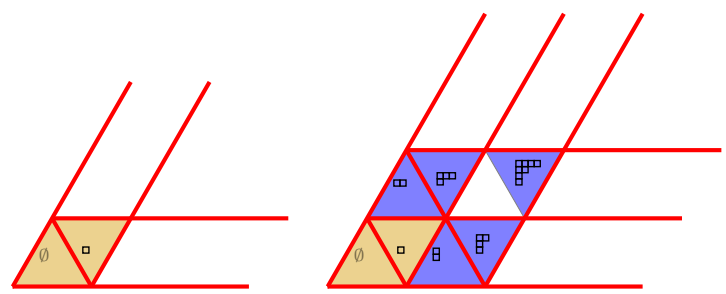

Fig. 2: $m$-maximal alcoves $w^{-1} \mathcal{A}_{0}$ in the dominant chamber of the $m$-Shi arrangement of type $A_{2}$, filled with the 3 -core partition $w \emptyset$. On the left $(m=1)$, they are also 2 -cores, and on the right $(m=2)$, they are also 5 -cores.

\subsection{Effect on m-minimal alcoves}

Define $\mathfrak{A}_{m}$ to be the $m$-dilation of $\mathcal{A}_{0}$ :

$$
\mathfrak{A}_{m}=\left\{v \in V \mid\left\langle v \mid \alpha_{i}\right\rangle \geq-m,\langle v \mid \theta\rangle \leq m+1\right\} .
$$

Note that the set of alcoves in $\mathfrak{A}_{m}$ is in bijection with $Q /(m n+1) Q$. Furthermore, it is easy to see by translating (by $m \rho=\frac{m}{2} \sum_{\alpha \in \Delta^{+}} \alpha$ ) that $Q \cap \mathfrak{A}_{m}$ is in bijection with $Q \cap \overline{(m n+1) \mathcal{A}_{0}}$. It is the latter that is discussed in Lemma 7.4.1 of Haiman (1994) and studied in Athanasiadis (2005) (technically for the co-root lattice $Q^{\vee}$ ). Taking the latter bijection into account, the second statement of Theorem 6.1 below appears in Theorem 4.2 of Athanasiadis (2005).

Theorem 6.1. 1. The map $w \mathcal{A}_{0} \mapsto w^{-1} \mathcal{A}_{0}$ restricts to a bijection between alcoves in the region $\mathfrak{A}_{m}$ and m-minimal alcoves.

2. The map $w(0, \ldots, 0) \mapsto w^{-1} \mathcal{A}_{0}$ restricts to a bijection between $Q \cap \mathfrak{A}_{m}$ and m-minimal alcoves in the dominant chamber.

Proof. Observe $\mathfrak{A}_{m}=H_{\theta, m+1}^{-} \cap \bigcap_{i=1}^{n-1} H_{\alpha_{i},-m}^{+}$can be viewed as an $m$-dilation of (the closure of) $\mathcal{A}_{0} \subseteq H_{\theta, 1}^{-} \cap \bigcap_{i=1}^{n-1} H_{\alpha_{i}, 0}^{+}$.

The second statement follows directly from Corollary 3.3 .

A proof of the first statement can be given that is very similar to that of Propositions 3.1 and 2.1 In Fishel and Vazirani (2010) we use those propositions to prove it.

The first part of the bijection is illustrated in Figures 3 and 4 , by comparing Figure 4 to Figure 3 

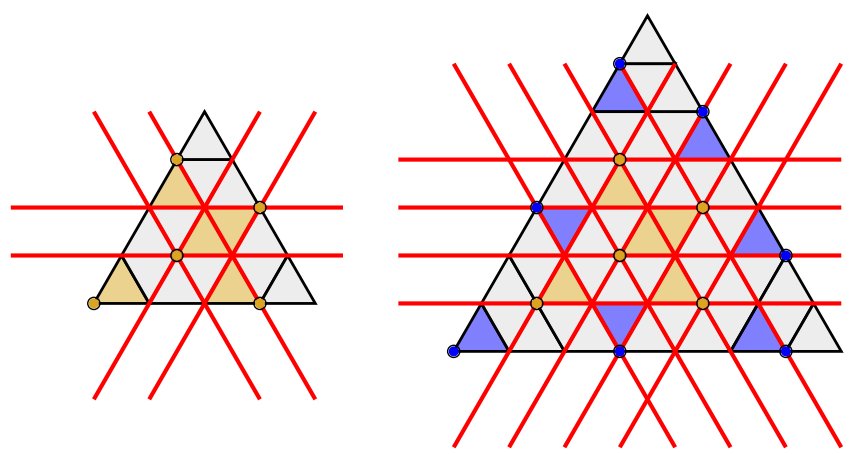

Fig. 3: $w \mathcal{A}_{0}$ for the $m$-minimal alcoves $w^{-1} \mathcal{A}_{0}$ in Figure 4 below, $m=1,2$. Note $w \mathcal{A}_{0} \subseteq \mathfrak{A}_{m}$. Each $\gamma \in Q$ is in precisely one yellow/blue alcove, so this illustrates the second statement of Theorem 6.1
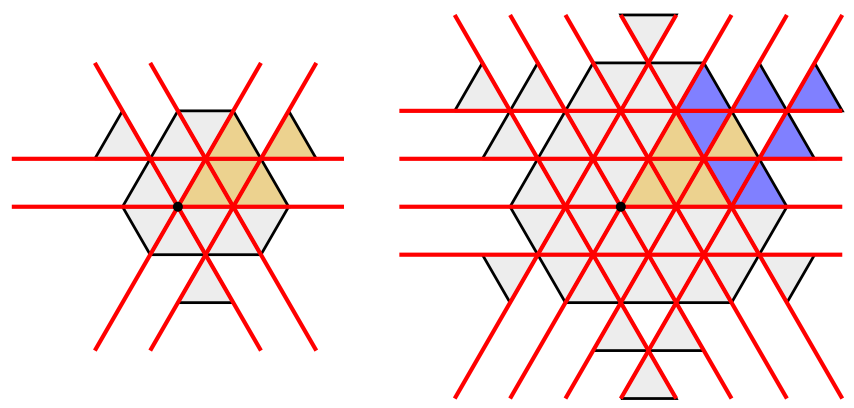

Fig. 4: $m$-minimal alcoves in the $m$-Shi arrangement for $m=1(m=2)$. Dominant alcoves are shaded yellow (and/or blue, respectively).

\subsection{Effect on m-maximal alcoves}

Let

$$
\mathfrak{a}_{m}=\left\{v \in V \mid\left\langle v \mid \alpha_{i}\right\rangle \leq m \text { for } 1 \leq i<n,\langle v \mid \theta\rangle \geq-m+1\right\}
$$

Theorem 6.2. $\quad$ 1. The map $w \mathcal{A}_{0} \mapsto w^{-1} \mathcal{A}_{0}$ restricts to a bijection between alcoves in the region $\mathfrak{a}_{m}$ and m-maximal alcoves.

2. The map $w(0, \ldots, 0) \mapsto w^{-1} \mathcal{A}_{0}$ restricts to a bijection between $Q \cap \mathfrak{a}_{m}$ and m-maximal alcoves in the dominant chamber.

The proof is similar to the proof of Theorem 6.1 .

The bijection is illustrated below, the first part comparing Figure 5 to Figure 6 , and the second part from restricting our attention to the lattice points. 

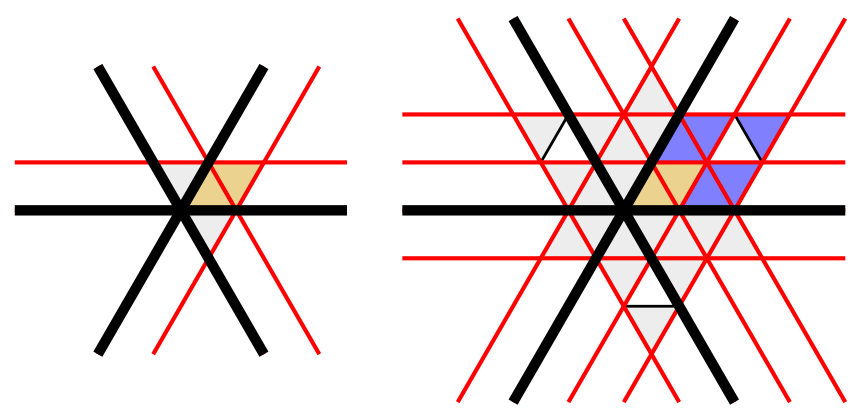

Fig. 5: $m$-maximal alcoves in the $m$-Shi arrangement for $m=1(m=2)$. Dominant alcoves are shaded yellow (and/or blue, respectively), whereas other $m$-maximal alcoves are shaded gray.
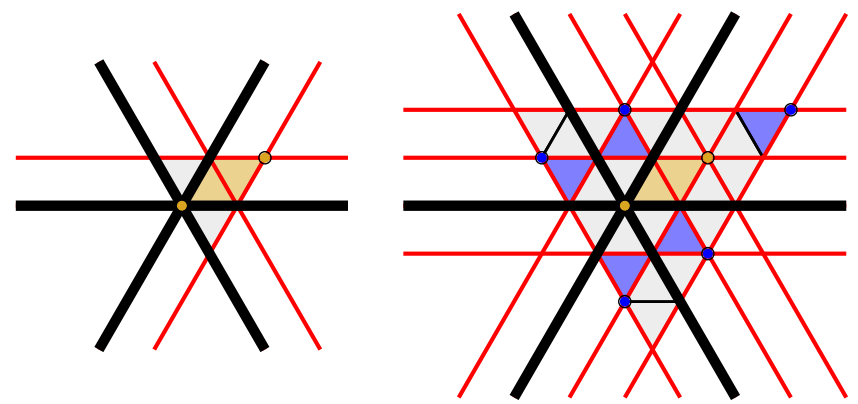

Fig. 6: $w \mathcal{A}_{0}$ for the $m$-maximal alcoves $w^{-1} \mathcal{A}_{0}$ in Figure 5 above, $m=1,2$. Note $\bigcup w \overline{\mathcal{A}_{0}}=\mathfrak{a}_{m}$. Each $\gamma=$ $w(0, \ldots, 0) \in Q \cap \mathfrak{a}_{m}$ is in precisely one yellow/blue alcove, so this illustrates the second statement of Theorem 6.2

\section{$7 \quad$ Alcove walls and addable and removable boxes for cores}

In this section, we show how certain alcove walls correspond to addable and removable boxes in cores. We characterize the regions counted by the Narayana numbers in terms of their corresponding cores and explain an analagous result for bounded regions.

We will use some ideas from the theory of crystal graphs. For those readers familiar with the realization of the basic crystal $B\left(\Lambda_{0}\right)$ of $\widehat{\mathfrak{s l}}_{n}$ as having nodes parameterized by $n$-regular partitions,

$$
s_{i} \lambda= \begin{cases}\tilde{f}_{i}^{\left\langle h_{i}, \mathrm{w} t(\lambda)\right\rangle}(\lambda) & \left\langle h_{i}, \mathrm{w} t(\lambda)\right\rangle \geq 0 \\ \tilde{e}_{i}^{-\left\langle h_{i}, \mathrm{w} t(\lambda)\right\rangle}(\lambda) & \left\langle h_{i}, \mathrm{w} t(\lambda)\right\rangle \leq 0\end{cases}
$$

where

$$
\mathrm{w} t(\lambda)=\Lambda_{0}-\sum_{(x, y) \in \lambda} \alpha_{y-x \bmod n}
$$

and $h_{i}$ is the co-root corresponding to $\alpha_{i}$.

Then the $n$-cores are exactly the $\widehat{\mathfrak{S}}_{n}$-orbit on the highest weight node, which is the empty partition $\emptyset$. 
It is well-known that $s_{i} \lambda=\mu$ iff $s_{i} \mathrm{w} t(\lambda)=\mathrm{w} t(\mu)$ where the action of $\widehat{\mathfrak{S}}_{n}$ on the weight lattice is given by

$$
s_{i}(\gamma)=\gamma-\left\langle\gamma \mid \alpha_{i}\right\rangle \alpha_{i} .
$$

We refer the reader to Chapters 5,6 of $\operatorname{Kac}(1990)$ for details on the affine weight lattice, definition of $\Lambda_{0}$ and so on. For computational purposes, all we need remind the reader of is that $\left\langle\Lambda_{0} \mid \alpha_{i}\right\rangle=\delta_{i, 0}$ and $\left\langle\alpha_{0} \mid \alpha_{i}\right\rangle=2 \delta_{i, 0}-\delta_{i, 1}-\delta_{i, n-1}$.

It is useful to recall the following notation from the theory of crystal graphs. In the case $s_{i}$ removes $k$ boxes of residue $i$ from the core $\lambda$, write $\varepsilon_{i}(\lambda)=k, \varphi_{i}(\lambda)=0$. In the case $s_{i}$ adds $r$ boxes to $\lambda$ to obtain $\mu$, write $\varepsilon_{i}(\lambda)=0, \varphi_{i}(\lambda)=r$.

\subsection{Narayana numbers}

In this section, we add another set to the list in Theorem 1.2 of Athanasiadis (2005) of combinatorial objects counted by generalized Narayana numbers. We further refine the enumeration of $n$-cores $\lambda$ which are also $(m n+1)$-cores. This refinement produces the $m$-Narayana numbers, or generalized Narayana numbers, $N_{n}^{m}(k)$, which are defined in Definition 7.4 below. Recall that the $(k, l)$-box of the $n$-core $\lambda$ is referred to as an $i$-box if it has residue $i=l-k \bmod n$. Our refinement here is to count the number of $n$-cores $\lambda$ which are also $(m n+1)$-cores by the number of residues $i$ such that $\lambda$ has exactly $m$ removable $i$-boxes.

Remark 7.1. Equation (7.1) says that if $s_{i}$ removes $k$ boxes (of residue i) from $\lambda$, or adds $-k$ boxes to $\lambda$ to obtain $\mu$, then $\mathrm{w} t(\mu)=s_{i}(\mathrm{w} t(\lambda))=\mathrm{w} t(\lambda)-k \alpha_{i}$. In either case, $\mathrm{w} t(\mu)=\mathrm{w} t(\lambda)+\left(\varphi_{i}(\lambda)-\varepsilon_{i}(\lambda)\right) \alpha_{i}$.

A straightforward rephrasing of Proposition 3.1 is then:

Proposition 7.2. Let $\lambda$ be an $n$-core, $k \in \mathbb{Z}_{>0}$, and $w \in \widehat{\mathfrak{S}}_{n}$ of minimal length such that $w \emptyset=\lambda$. Fix $0 \leq i<n$. The following are equivalent:

1. $\lambda$ has $k$ many removable $i$-boxes; in particular $\left|s_{i} \lambda\right|=|\lambda|-k$ as the action of $s_{i}$ removes those $i$-boxes.

2. $\left\langle\vec{n}(\lambda) \mid \alpha_{i}\right\rangle=-k$ for $i \neq 0, \quad\langle\vec{n}(\lambda) \mid \theta\rangle=k+1$ for $i=0$,

3. $w^{-1} \mathcal{A}_{0} \subseteq H_{\alpha, k}^{+}, \quad w^{-1} s_{i} \mathcal{A}_{0} \subseteq H_{\alpha, k}^{-}$where $w^{-1}\left(\alpha_{i}\right)=\alpha-k \delta$.

When we rephrase Corollary 3.3 in this context, it says:

Proposition 7.3 (Corollary 3.3 restated). Suppose $\lambda=w \emptyset$ is the $n$-core associated to the dominant alcove $\mathcal{A}=w^{-1} \mathcal{A}_{0}$ via the bijection $\Phi$ of Section 5 .

Then $\mathcal{A}$ is m-minimal if and only if whenever $\lambda$ has exactly $k$ removable boxes of residue $i$ then $k \leq m$. (And in this case, $\lambda$ is also an $(m n+1)$-core.)

\subsubsection{A refinement}

Proposition 7.2 thus gives us another combinatorial interpretation of the $m$-Narayana numbers, as in Athanasiadis (2005).

Definition 7.4. The $k^{\text {th }} m$-Narayana number of type $A$ is

$$
N_{n}^{m}(k)=\frac{1}{n m+1}\left(\begin{array}{c}
n-1 \\
n-k-1
\end{array}\right)\left(\begin{array}{c}
m n+1 \\
n-k
\end{array}\right) .
$$


Recall from Athanasiadis (2005) that $N_{n}^{m}(k)$ is the number of dominant regions of the $m$-Shi arrangement which have exactly $k$ hyperplanes $H_{\alpha, m}$ separating them from $\mathcal{A}_{0}$ such that $H_{\alpha, m}$ contains a wall of the region.

In other words, for fixed $k$, we count how many $m$-minimal alcoves $\mathcal{A}=w^{-1} \mathcal{A}_{0}$ satisfy that for exactly $k$ positive roots $\alpha$, there exists an $i$ such that $w^{-1} \mathcal{A}_{0} \subseteq H_{\alpha, m}^{+}$but $w^{-1} s_{i} \mathcal{A}_{0} \subseteq H_{\alpha, m}^{-}$. It is clear that

$$
\sum_{k \geq 0} N_{n}^{m}(k)=m \text {-Catalan number }
$$

since each dominant $m$-minimal alcove gets counted once.

By Proposition 7.2 above, $N_{n}^{m}(k)$ equivalently counts how many $n$-cores $\lambda$ that are also $(m n+1)$-cores have exactly $k$ distinct residues $i$ such that $\lambda$ has precisely $m$ removable $i$-boxes.

Corollary 7.5. Let $N_{n}^{m}(k)$ denote the $m$-Narayana number of type $A_{n-1}$. Then

$$
\begin{gathered}
N_{n}^{m}(k)=\mid\{\lambda \mid \lambda \text { is an } n \text {-core and }(m n+1) \text {-core and } \exists K \subseteq \mathbb{Z} / n \mathbb{Z} \\
\text { with }|K|=k \text { such that } \lambda \text { has exactly } m \text { removable boxes } \\
\text { of residue } i \text { iff } i \in K\} \mid .
\end{gathered}
$$

\subsection{Bounded regions}

We rephrase Equation (7.1) and Proposition 3.1 again, this time for $m$-maximal alcoves and bounded regions.

Recall Remark7.1. In the context of $m$-maximal alcoves and bounded regions, Proposition 3.1 becomes

Proposition 7.6. Let $\lambda$ be an $n$-core, $k \in \mathbb{Z}_{>0}$, and $w \in \widehat{\mathfrak{S}}_{n}$ of minimal length such that $w \emptyset=\lambda$. Fix $0 \leq i<n$. The following are equivalent

1. $\varphi_{i}(\lambda)=k$,

2. $\left\langle\vec{n}(\lambda) \mid \alpha_{i}\right\rangle=k$ for $i \neq 0, \quad\langle\vec{n}(\lambda) \mid \theta\rangle=-k+1$ for $i=0$,

3. $w^{-1} \mathcal{A}_{0} \subseteq H_{\alpha, k}^{-}, \quad w^{-1} s_{i} \mathcal{A}_{0} \subseteq H_{\alpha, k}^{+}$where $w^{-1}\left(\alpha_{i}\right)=-\alpha+k \delta$.

When we rephrase Corollary 3.4 in this context, it says:

Suppose $\lambda=w \emptyset$ is the $n$-core associated to the dominant alcove $\mathcal{A}=w^{-1} \mathcal{A}_{0}$. Then $\mathcal{A}$ is $m$-maximal iff whenever $\lambda$ has exactly $k$ addable boxes of residue $i$ then $k \leq m$. (And in this case, $\lambda$ is also an $(m n-1)$-core.)

As a consequence, note an $n$-core $\lambda$ is automatically an $(m n-1)$-core if $\varphi_{i}(\lambda) \leq m$ for all $0 \leq i<n$. Athanasiadis and Tzanaki (2006) define $h_{k}^{+}(\Delta, m), 0 \leq k<n$ as the number of bounded dominant regions of $\mathcal{S}_{n}^{m}$ for which exactly $n-1-k$ hyperplanes of the form $H_{\alpha, m}, \alpha \in \Delta^{+}$are walls (i.e. support a facet) of that region and do not separate it from the fundamental alcove $\mathcal{A}_{0}$.

By the definition of $m$-maximal, we can replace a bounded region by its unique $m$-maximal alcove and consider its walls instead. In other words, to calculate $h_{k}^{+}(\Delta, m)$, we count how many $m$-maximal alcoves $\mathcal{A}=w^{-1} \mathcal{A}_{0}$ satisfy that for exactly $n-1-k$ positive roots $\alpha$, there exists an $i$ such that $w^{-1} \mathcal{A}_{0} \subseteq H_{\alpha, m}^{-}$ but $w^{-1} s_{i} \mathcal{A}_{0} \subseteq H_{\alpha, m}^{+}$.

By Proposition 7.6 above, $h_{k}^{+}(\Delta, m)$ equivalently counts how many $n$-cores $\lambda$ that are also $(m n-1)$ cores have exactly $n-1-k$ distinct residues $i$ such that $\lambda$ has precisely $m$ addable $i$-boxes. 


\section{Acknowledgements}

Both authors wish to thank AIM and the SQuaREs program where this work was started. We thank Nathan Reading who pointed out that the number of bounded dominant regions in the complement of the $m$-Shi arrangement is counted by $\frac{1}{s+t}\left(\begin{array}{c}s+t \\ t\end{array}\right)$ for appropriate $s, t$, and by Anderson (2002) this number also counts the number of partitions that are $s$-cores as well as $t$-cores. We thank Mark Haiman for pointing out parts of Section 6 to us.

\section{References}

J. Anderson. Partitions which are simultaneously $t_{1}$ - and $t_{2}$-core. Discrete Math., 248(1-3):237-243, 2002. ISSN 0012-365X.

C. A. Athanasiadis. On a refinement of the generalized Catalan numbers for Weyl groups. Trans. Amer. Math. Soc., 357(1):179-196 (electronic), 2005. ISSN 0002-9947.

C. A. Athanasiadis and E. Tzanaki. On the enumeration of positive cells in generalized cluster complexes and Catalan hyperplane arrangements. J. Algebraic Combin., 23(4):355-375, 2006. ISSN 0925-9899.

C. Berg, B. Jones, and M. Vazirani. A bijection on core partitions and a parabolic quotient of the affine symmetric group. J. Combin. Theory Ser. A, 116(8):1344-1360, 2009. ISSN 0097-3165.

S. Fishel and M. Vazirani. A bijection between bounded dominant Shi regions and core partitions, 2009.

S. Fishel and M. Vazirani. A bijection between dominant Shi regions and core partitions. European J. Combin., 2010. doi: 10.1016/j.ejc.2010.05.014.

F. Garvan, D. Kim, and D. Stanton. Cranks and t-cores. Invent. Math., 101(1):1-17, 1990. ISSN 00209910.

M. D. Haiman. Conjectures on the quotient ring by diagonal invariants. J. Algebraic Combin., 3(1):17-76, 1994. ISSN 0925-9899.

G. James and A. Kerber. The representation theory of the symmetric group, volume 16 of Encyclopedia of Mathematics and its Applications. Addison-Wesley Publishing Co., Reading, Mass., 1981. ISBN 0-201-13515-9. With a foreword by P. M. Cohn, With an introduction by Gilbert de B. Robinson.

V. G. Kac. Infinite-dimensional Lie algebras. Cambridge University Press, Cambridge, third edition, 1990. ISBN 0-521-37215-1; 0-521-46693-8.

A. Lascoux. Ordering the affine symmetric group. In Algebraic combinatorics and applications (Gößweinstein, 1999), pages 219-231. Springer, Berlin, 2001.

J. Y. Shi. Alcoves corresponding to an affine Weyl group. J. London Math. Soc. (2), 35(1):42-55, 1987. ISSN 0024-6107. 